\title{
CURRENT PLATE MOTIONS
}

\author{
Richard Gordon, Charles DeMets, Seth Stein, Don Argus, and Dale Woods: \\ Department of Geological Sciences, Northwestern University, Evanston, Il- \\ linois 60201
}

The standard against which VLBI measurements of continental drift and plate motions are cumpared are self-consistent global models of "present-day" plate motions determined from geophysical data: marine magnetic anomalies at oceanic spreading centers, azimuths of transform faults, and orientations of earthquake slip vectors on transform faults and at subduction zones. Past global plate motion models have defined regions where the assumption that plates behave rigidly has apparently lead to systematic misfits, sometimes exceeding $10 \mathrm{~mm} / \mathrm{yr}$, of plate motion data. Here, we present some of the results from NUVEL-1, a new, self-consistent global model of present-day relative plate motions determined from a compilation and analysis of existing and new geophysical data. These data and new techniques have allowed us to eliminate nearly all statistically significant systematic misfits identified by earlier models, suggesting that the rigidplate assumption is an excellent approximation when plate motions are averaged over several million years. Beside improving estimates of the motion on previously identified plate boundaries, we have also identified and determined motions on other boundaries whose subtle morphologies, lack of seismicity, and very slow $(<10 \mathrm{~mm} / \mathrm{yr})$, relative motions have made them difficult to detect. Here we focus on the application of VLBI measurements to help resolve plate tectonic problems and then briefly outline our results for Pacific-North America motion and plate motions in the Indian Ocean.

What important plate tectonic problems can VLBI measurements address? Since the NUVEL-1 model, like earlier models, averages plate motions over $3 \mathrm{Ma}$, it has nothing to say about short-term plate motion episodicity, something that geodetic techniques such as VLBI can measure directly. Second, conventional geophysical data can directly determine the motion only between two plates which share a boundary. Estimates of motion between plates lacking a common boundary or separated by a subduction zone can only be made by invoking plate circuit vector closure constraints based on the assumption that the plates are rigid (see Figure 1). In contrast, VLBI measurements can yield the rate and direction of motion between any two plates. Comparison between rates measured by VLBI to those predicted by closure of the plate motion model at subduction zones and between plate pairs without a common boundary thus tests the extent to which plates act rigidly.

Plate motion models have long suggested that the rate of motion between the Pacific and North American plates is $\sim 60 \mathrm{~mm} / \mathrm{yr}$, in contrast to the well-documented $\sim 35 \mathrm{~mm} / \mathrm{yr}$ of motion along the San Andreas fault. This discrepancy, termed the San Andreas discrepancy, presumably must be reconciled by extension in the Basin and Range and by strike-slip motion on other California faults parallel to the San Andreas. However, estimates of the rate and direction of Basin and Range extension fall at least 
$10 \mathrm{~mm} / \mathrm{yr}$ short of the expected Pacific-North America rate [Minster and Jordan, 1984]. A compilation and analysis of the available marine magnetic profiles from the southern Gulf of California, where the long-term measure of the Pacific-North America rate can be found, suggests spreading in the Gulf has averaged $48 \mathrm{~mm} / \mathrm{yr}$ since $3 \mathrm{Ma}, 20 \%$ lower than estimates of the Gulf spreading rate [Larson et al., 1968] used in prior models [e.g. Chase, 1978, Minster and Jordan, 1978].

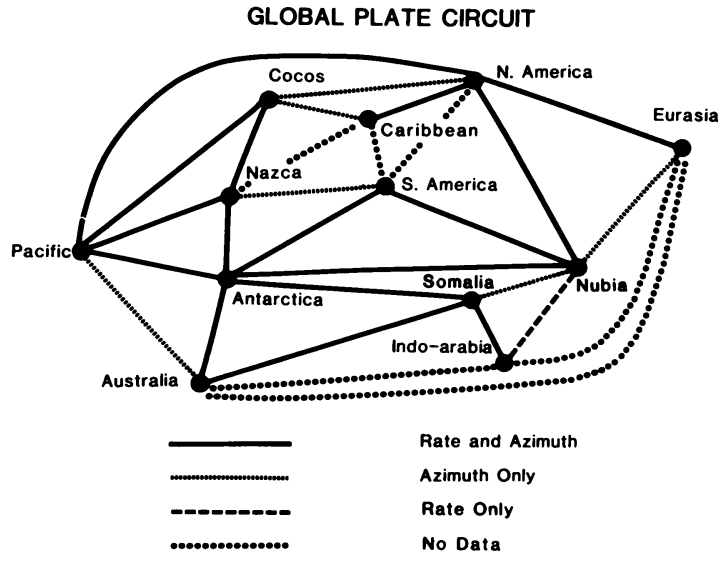

Figure 1

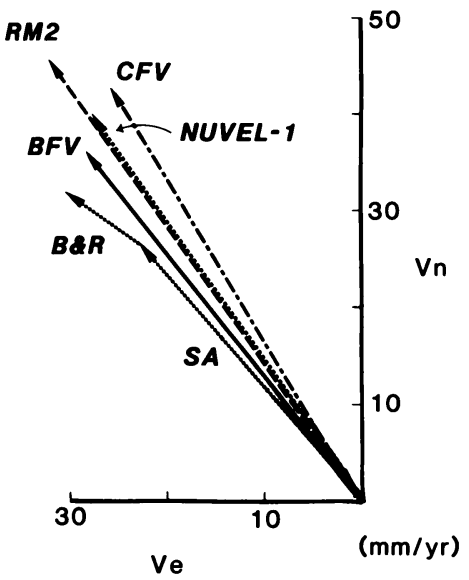

Figure 2

Independently, when direct Pacific-North America data are deleted from the NUVEL-1 global dataset, we predict rates of $49 \mathrm{~mm} / \mathrm{yr}$ in the southern Gulf and in central California, implying that the "missing motion" along the San Andreas is $30 \%$ less than estimated from prior models. Figure 2 shows that the observed relative motions across the San Andreas Fault and the Basin and Range province fail to sum to the Pacific-North America velocity vector predicted by NUVEL-1. Both NUVEL-1 and Minster and Jordan's [1.978] RM2 model predict comparable ( $\sim 9 \mathrm{~mm} / \mathrm{yr})$ shortening across the plate boundary zone; however, NUVEL-1 predicts only $4 \mathrm{~mm} / \mathrm{yr}$ of additional slip parallel to the San Andreas, $\sim 60 \%$ less than predicted by RM2.

The Indian Ocean plate motion circuit between the Antarctic, African, and Australian plates has posed problems for prior global relative plate motion models and therefore provides an important test of the rigicl-plate hypothesis. The Indian Ocean is of ten cited as the type-location of oceanic intraplate deformation because of the nonclosure found in prior models, high levels of seismicity and heat flow, mappable reverse faults, and undulations in topography and gravity. The three spreading centers, the Central, Southwest, and Southeast Indian ridges are all long and have well-distributed observations of azimuths and rates, thereby guaranteeing strong constraints on the individual Euler vectors and a strong test of circuit closure. Our evaluation of Indian Ocean plate circuit closure [DeMets et al., 1987], using a statistical test of closure [Gordon et al., 1987], yields no evidence for non-rigid plate behavior at even the $2-3 \mathrm{~mm} / \mathrm{yr}$ level, a result mainly attributable to new spreading rate data from the Southeast Indian Ridge and a revised geometry for the Indian and Arabian plates [Wiens et al., 1985]. In contrast to earlier models, we place India and Australia on separate plates and predict the distance between the continents is decreasing at $12 \mathrm{~mm} / \mathrm{yr}$. This could be tested using VLBI between stations in India and Australia. 